\title{
Systematics of Staggered Fermion Spectral Properties and Topology
}

\author{
Kit Yan Wong \\ Simon Fraser University, Department of Physics, \\ 8888 University Drive, Burnaby, British Columbia, Canada V5A 1 S6 \\ R. M. Woloshyn \\ TRIUMF, 4004 Wesbrook Mall, Vancouver, British Columbia, Canada V6T 2A3
}

\begin{abstract}
The spectral properties of a variety of improved staggered operators are studied in quenched QCD. The systematic dependence of the infrared eigenvalue spectrum on i) improvement in the staggered operator, ii) improvement in the gauge field action, iii) lattice spacing and iv) lattice volume, is analyzed. It is observed that eigenmodes with small eigenvalues and large chirality appear as the level of improvement increases or as one approaches the continuum limit. These eigenmodes can be identified as the "zero modes" which contribute to the chirality associated, via the index theorem, with the topology of the background gauge field. This gives evidence that staggered fermions are sensitive to gauge field topology. After successfully identifying these would-be chiral zero modes, the distribution of the remaining non-chiral modes is compared with the predictions of Random Matrix Theory in different topological sectors. Satisfactory agreement is obtained.
\end{abstract}

\section{INTRODUCTION}

It is well known that spectral flow of the massless Dirac operator $(D)$ is related to the topology of the background gauge fields. When massless fermions are coupled to Yang-Mills fields with non-trivial topology, one or more eigenvalues of the corresponding Dirac operator necessarily vanish. This is formally given by the Atiyah-Singer index theorem 1]

$$
Q=\operatorname{index}(D) \equiv n_{L}-n_{R},
$$

where $\mathrm{Q}$ is the topological charge of the gauge field and $n_{L}\left(n_{R}\right)$ are the numbers of left(right) zero eigenmodes of the Dirac operator.

On the other hand, until recently it was believed that staggered fermions do not feel gauge field topology [2] because of the lack of zero eigenmode of the operator at finite lattice spacing. This unpleasant feature of staggered fermions was further revealed in comparisons of the infrared eigenvalue spectrum obtained in simulations with the predictions of Random Matrix Theory (RMT) [3, 4, 5]. The eigenvalue spectrum in all topological charge sectors was found to be consistent with the prediction of RMT for topological charge equal to zero [4, 5].

In the past year this problem has been revisited and the situation has changed. Recent calculations $6,7,8$ show that with staggered operator improvement for staggered fermions the correct response to QCD topology, governed by the index theorem, can be obtained. ${ }^{1}$ In [8] we presented some of our results in a preliminary form. Here we give our final results along with the study of some systematic effects not included in our earlier work.

\footnotetext{
${ }^{1}$ Earlier Farchioni et al. [9] suggested that in the Schwinger model topology may be visible in the spectrum of the staggered Dirac operator.
}

The failure of unimproved staggered fermion actions to show the proper topological features is due to lattice artifacts, in particular flavour-changing interactions. This was already suggested in Ref. [10]. The staggered quark action describes four quark flavours in the continuum limit and the eigenvalue spectrum has a 4-fold degeneracy in this limit. At finite lattice spacing, the flavour-changing interactions break the flavour symmetry and the degeneracy is lifted. Consequently, staggered fermions do not have exact zero modes at finite lattice spacing because the continuum chiral modes (if there are any) are scattered on the lattice. One thus expects staggered fermions to show better topological properties if flavour-changing effects can be suppressed.

In addition to lattice artifacts, the spectral density in the infrared limit also depends sensitively on the volume of the lattice. This can be seen from the fact that, because the topological susceptibility defined by $\left\langle Q^{2}\right\rangle / V$ is independent of the volume $V$, the ensemble average $\left\langle Q^{2}\right\rangle$ scales approximately with $V$. For example, previous studies [11, 12] on the spectral properties of the overlap operator have revealed this strong finite volume effect (in contrast with the staggered Dirac operator, the overlap fermion operator [14] has exact chiral symmetry 15] on the lattice and has true zero modes even at finite lattice spacing, see section III). In particular, it has been shown that satisfactory agreement with the predictions of RMT can be achieved only when the physical volume of the lattice exceeds $(1.2 \mathrm{fm})^{4}$. On smaller volumes the agreement is less appealing and the sensitivity to topology disappears.

Both of these issues have been examined in this project by studying the spectral flow in the infrared limit for a variety of improved staggered operators. The improved staggered operators are designed to suppress flavourchanging interactions by smoothing out the quark-gluon interaction vertex [16]. Four different sources of systematic effects have been considered: 
- improvement in the staggered operator

- improvement in the gauge field action

- lattice spacing

- lattice volume.

Simulations are done both with the standard Wilson gluon action and the $\mathcal{O}\left(a^{2} \alpha_{s}\right)$ accurate tadpole improved gluon action [17] for a range of lattice spacings and volumes. It is observed that eigenmodes with small eigenvalues and large chirality appear as the level of improvement increases $[6,[7,[8]$. These small eigenmodes can be identified as the chiral zero modes associated with the topology of the background gauge fields. Our results also show the expected strong dependence on volume. Only a few "zero modes" are observed when the physical volume is small and the number increases as the volume increases.

The paper is organized as follows. The improved staggered operators and their properties will be reviewed in the next section. A similar review on the overlap operator is given in section [III Details of the simulations are discussed in section IV and results will be presented in section $\nabla$ After successfully identifying the "zero modes", distribution of the remaining non-chiral modes is compared with the predictions of RMT in Section VI

\section{IMPROVED STAGGERED OPERATORS}

The unimproved staggered Dirac operator is

$$
D^{s}(x, y)=\frac{1}{2} \sum_{\mu} \eta_{\mu}(x)\left[U_{\mu}(x) \delta_{x+\hat{\mu}, y}-U_{\mu}^{\dagger}(y) \delta_{x-\hat{\mu}, y}\right]
$$

where $\eta_{\mu}(x)=(-1)^{x_{1}+\ldots+x_{\mu-1}}$ is the standard staggered fermion phase. A variety of improvement schemes are considered here. The basic improved version is the $\mathcal{O}\left(a^{2}\right)$ tree-level improved Asq operator [18, which includes an additional 3-link Naik term and replaces the gauge field in (2) with Fat7 effective links (sum of the original link and the nearby paths, up to 7-link staples). Tadpole improvement of the Asq operator gives the $\mathcal{O}\left(a^{2} \alpha_{s}\right)$ accurate Asqtad operator. This is the staggered fermion operator which has been used in recent dynamical fermion simulations [19].

It has been found that there are alternate improvement schemes that can suppress flavour-symmetry breaking lattice artifacts even more than Asq improvement. One such scheme is based on unitarized Fat7 (UFat7) links. The usefulness of UFat7 smearing was first discussed by Lee 20] and a staggered action which iterates this fattening procedure giving the improved Asq operators $(\mathrm{UFat7})^{n} \times$ Asq was studied by Follana et al. [21]. The HYP-improved operators (HYP) ${ }^{n}$ [22] are constructed from unitarized fat links also but only those links within the hypercubes containing the original link are included in the fattening process. Readers are referred to the respective papers for further details.
The staggered Dirac operator is anti-Hermitian and since $\left\{D^{s}, \epsilon(x)\right\}=0$ where $\epsilon(x)=(-1)^{x_{1}+\ldots+x_{4}}$, the eigenvalues of $D^{s}$ are pure imaginary and come in pairs $\pm i \lambda$. The commutation relation $\left\{D^{s}, \epsilon(x)\right\}=0$ is a remnant of the continuum global chiral symmetry on the lattice. In addition, it is well known that $\left(D^{s}\right)^{2}$ connects only even-even $\left(\left(D_{e e}^{s}\right)^{2}\right)$ or odd-odd $\left(\left(D_{o o}^{s}\right)^{2}\right)$ sites on the lattice. In this project, we choose to compute the eigenvalues of $\left(D_{e e}^{s}\right)^{2}$ in favour of $D^{s}$ since it is Hermitian and has real eigenvalues $\lambda^{2}$. Note that the extra doubling of modes induced by squaring is canceled by working only on even sites of the lattice. To be precise, we compute the lowest 40 eigenvalues of $\left(D_{e e}^{s}\right)^{2}$ which correspond to the lowest 40 positive (imaginary) eigenvalues of $D^{s}$.

Before leaving this section it is useful to understand how the low-lying non-chiral modes scale with the various systematics. This explains the scales used in the spectral plots given later in section D. In Ref. [13], Kalkreuter computed the complete spectrum of the unimproved staggered Dirac operator on $12^{4}$ lattices at several lattice spacings. Results show an accumulation of small eigenvalues with increasing lattice spacing. In addition, the number of eigenvalues increases when the volume of the lattice increases. Consequently, the magnitude of the non-zero eigenvalues in the infrared limit decreases with increasing lattice spacing and volume. On the other hand, when lattice spacing decreases, i.e., one goes closer to the continuum limit, an increase in the magnitude of the low-lying non-chiral modes is expected. The same should happen as we come closer to the continuum by improving the action. These scaling properties will be reflected in the choice of axes for the spectral graphs.

\section{THE OVERLAP OPERATOR}

The massless overlap operator is given by 14

$$
D^{o}=1+\gamma_{5} \epsilon\left(H^{w}\right)
$$

where $\epsilon\left(H^{w}\right)$ is the matrix sign function

$$
\epsilon\left(H^{w}\right)=\frac{H^{w}}{\left|H^{w}\right|},
$$

and $H^{w}=\gamma_{5} D^{w}$ is related to the standard Wilson Dirac operator

$$
\begin{aligned}
D_{a \alpha, b \beta}^{w}(x, y) & =\delta_{a b} \delta_{\alpha \beta} \delta_{x, y} \\
& -\kappa \sum_{\mu}\left[\left(1-\gamma_{\mu}\right)_{\alpha \beta} U_{\mu}(x)_{a b} \delta_{x, y-\hat{\mu}}\right. \\
& \left.+\left(1+\gamma_{\mu}\right)_{\alpha \beta} U_{\mu}^{\dagger}(y)_{a b} \delta_{x, y+\hat{\mu}}\right] .
\end{aligned}
$$

The hopping parameter $\kappa$ is related to the bare mass $m$ by

$$
\kappa=\frac{1}{-2 m+8} .
$$




\begin{tabular}{|c|c|c|c|c|}
\hline$\beta$ & Action & $a^{2} \sigma$ & $a(\mathrm{fm})$ & Volume $V$ \\
\hline 5.85 & Wilson & $0.0748(7)$ & 0.123 & $10^{4}$ \\
\hline 6.0 & Wilson & $0.0478(5)$ & 0.0981 & $12^{4}$ \\
\hline 6.2 & Wilson & $0.0259(5)$ & 0.0722 & $16^{4}$ \\
\hline 8.26 & improved & $0.0724(5)$ & 0.121 & $8^{4}, 10^{4}, 12^{4}, 16^{4}$ \\
\hline 8.62 & improved & $0.0456(4)$ & 0.0958 & $12^{4}$ \\
\hline 9.18 & improved & $0.0246(5)$ & 0.0704 & $16^{4}$ \\
\hline
\end{tabular}

TABLE I: Simulation parameters and measured string tensions $a^{2} \sigma$. The couplings are chosen such that lattice spacings agree between simulations using the Wilson gluon action and the improved gauge field action.

A detail description of how $\kappa$ should be chosen is given in 23. We use $\kappa=0.21$ or $m=1.62$, which has been shown to be appropriate for our study of topology [12].

The overlap operator (3) satisfies the Ginsparg-Wilson relation [24]

$$
\left\{\gamma_{5}, D^{o}\right\}=D^{o} \gamma_{5} D^{o}
$$

which has exact chiral symmetry on the lattice. Consequently in contrast with the staggered Dirac operator, the overlap operator has true zero modes even at finite lattice spacing. The non-chiral modes come in complex conjugate pairs. Again, we use the operator $D^{o \dagger} D^{o}$ which is Hermitian and positive definite and compute the lowest 5 eigenvalues in each chiral sector. The matrix sign function $\epsilon\left(H^{w}\right)$ is approximated by a 14th order Zolotarev expansion [25]

$$
\epsilon\left(H^{w}\right) \simeq H^{w} \cdot \sum_{i=1}^{14} \frac{c_{i}}{H^{w^{\dagger}} H^{w}+b_{i}}
$$

with maximum errors $\sim \mathcal{O}\left(10^{-10}\right)$ in the interval $[0.04,1.5]$.

\section{SIMULATIONS}

Simulations are done with both the Wilson gluon action and the tadpole improved gauge field action for a range of lattice spacings (or couplings) and volumes. The physical scale is defined through the quenched string tension $\sqrt{\sigma} \simeq 0.44 G e V$. A new smearing method using unitarized Fat7 links [26] is used in the computation of the Wilson loops which substantially reduces the statistical fluctuations in the confining potential. Simulation parameters and the measured string tensions $a^{2} \sigma$ are listed in Table【 The couplings were chosen carefully such that lattice spacings agree between simulations using the Wilson gluon action and the improved gauge field action. A total of 1000 configurations were generated in each case.
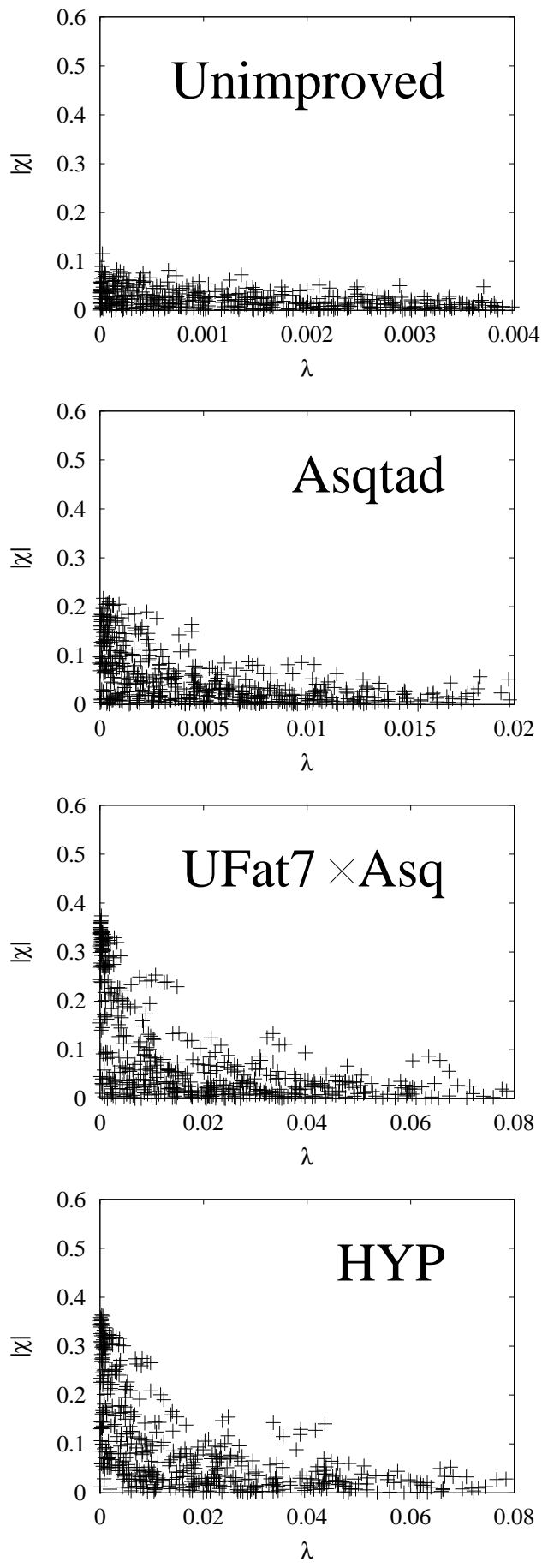

FIG. 1: Spectral graphs for the different operators where chirality $|\chi|$ is plotted against the eigenvalue $\lambda$. The ensemble is generated by the standard Wilson action at $\beta=5.85$ $(a \simeq 0.123 \mathrm{fm})$ on $10^{4}$ lattice. Results are shown for 50 configurations. Note that the scale for $\lambda$ increases when the level of improvement increases. 


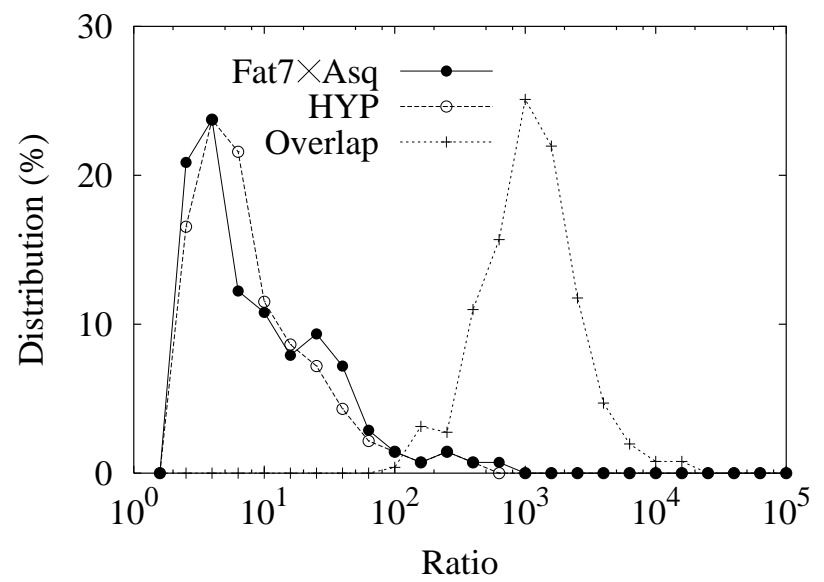

FIG. 2: Distribution of the ratios of eigenvalues between the smallest non-chiral mode and the largest "zero mode" for configurations with non-trivial topology. Results are shown for unimproved gauge fields at $\beta=5.85(a \simeq 0.123 \mathrm{fm})$ and lattice volume $V=10^{4}$.

\section{RESULTS}

\section{A. Staggered Operator Improvement}

We first examine the effect of staggered operator improvement on the infrared eigenvalue spectrum. Comparisons are done with unimproved gauge fields for $\beta=5.85$ $(a \simeq 0.123 \mathrm{fm})$ and $V=10^{4}$. The same comparisons with improved gauge fields (same lattice spacing and volume) will be presented in the next section. Results are shown in Fig. 1 where chirality $|\chi|$ is plotted against the eigenvalue $\lambda$ for the different operators. Note that the chirality of an eigenmode is given by the expectation value of $\gamma_{5}$, which is a 4 -link operator in the staggered basis 27]. The same scale is used for the chirality. It can be observed that the eigenvalue spectrum depends quite sensitively on the way in which the staggered Dirac operator is improved. Eigenmodes with small eigenvalues and relatively large chirality appear as the level of improvement increases. These small eigenmodes are taken to be the chiral "zero modes" which contribute to the index associated with the topology of the background gauge fields $5,10,28$. They can be identified by their separation in energy and chirality from the rest of the spectrum (see also, Ref. [6]). Throughout this project the following criteria are used for an eigenstate to be identified as a "zero mode": i) it is at least a factor of two smaller in eigenvalue than the smallest non-chiral mode and ii) the chirality is at least five times larger than that of the smallest non-chiral mode.

One can see also that as the level of improvement increases, the continuum fourfold degeneracy emerges where the scattered eigenmodes begin to form quartets. Note that at this lattice spacing with unimproved gauge fields the Asqtad operator is not sensitive to the topology and lattice artifacts are still dominant. One only starts to

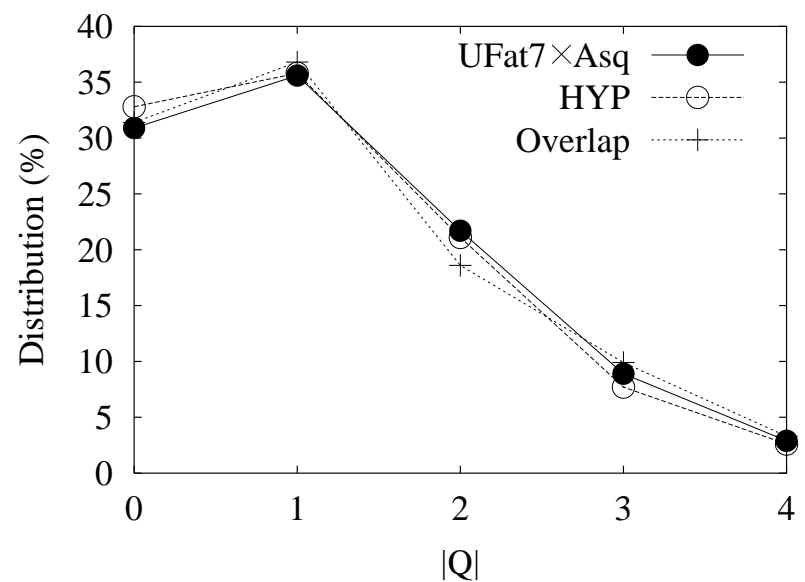

FIG. 3: Topological charge distributions (as a percentage of configurations) obtained by using different operators (unimproved gauge fields, $\beta=5.85(a \simeq 0.123 \mathrm{fm})$ and $\left.V=10^{4}\right)$.

see a separation between the "zero modes" and the nonchiral modes with further improved operators. In addition, a large renormalization is observed for the chirality of the would-be zero modes, which is $|\chi| \sim 0.5$ instead of unity. This large renormalization was also observed in previous studies 10, 29]. Nevertheless, the "zero modes" can be identified without any difficulty in most of the cases for the UFat7 $\times$ Asq and HYP operators.

To quantify the separation between the "zero modes" and the non-chiral modes, the distribution of the ratios of eigenvalues between the smallest non-chiral mode and the largest "zero mode" is plotted in Fig. [2 for configurations with non-trivial topology (i.e., "zero modes" exist). We should mention that the configurations in Fig. 2] are different for different operators as the topological indices obtained by using different operators do not always agree on a configuration by configuration basis (see next paragraph). It is possible that for a given configuration there are no chiral modes for one operator while they exist for the others. For comparison results of the overlap operator are also shown. Theoretically, the ratio is infinite for the overlap operator because exact zero modes exist on the lattice for overlap fermions. It is finite here solely because of computational precision. Results here show that the staggered operators are less sensitive to the topology at this lattice spacing with unimproved background gauge fields. The ratios are always three orders of magnitude for the overlap operator but the distribution is peaked at a ratio $\simeq 5$ for the staggered Dirac operators. Nevertheless about $85 \%$ of the configurations have ratios $\geq 5$ which allows the "zero modes" to be identified. In addition our results also show that improvements using UFat7 links or hyper-cubic blocking are equally efficient.

The identification of the topological charge index for a given gauge filed configuration is not unique. Because of lattice artifacts and the different ways the staggered operator is improved, it can be expected that the topolog- 

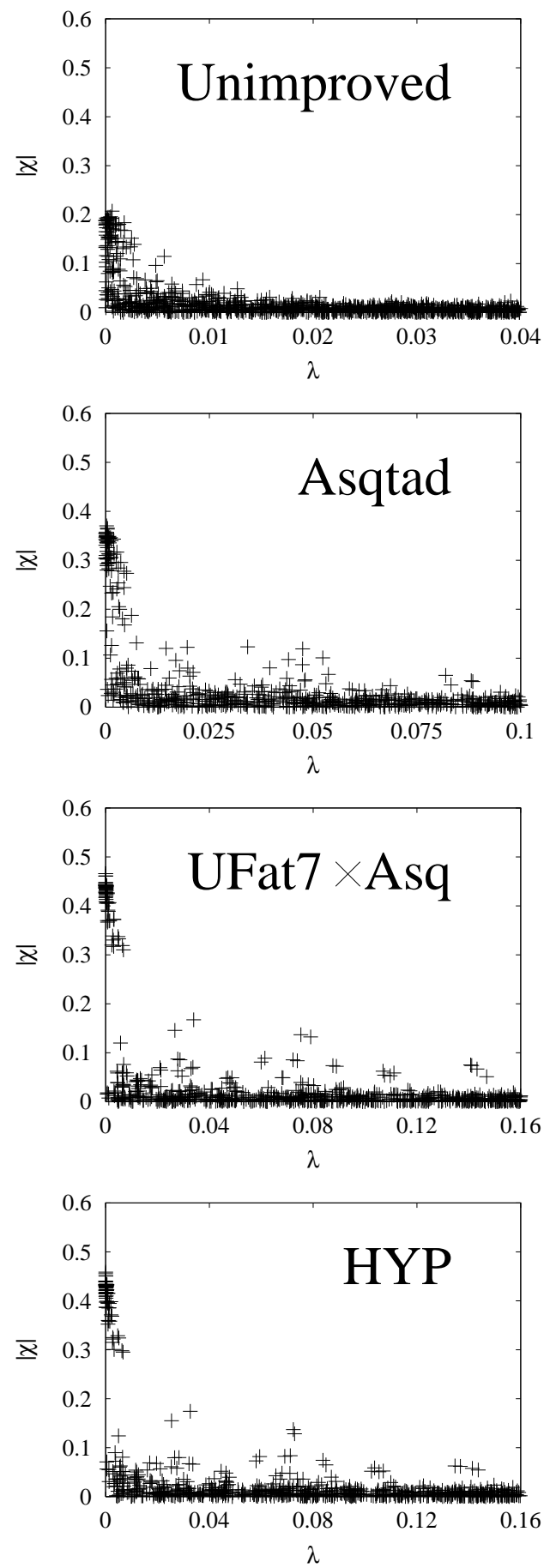

FIG. 4: Same as Fig. 1 but with improved gauge field action $\left(\beta=8.26(a \simeq 0.121 \mathrm{fm}), V=10^{4}\right)$. Note that the scale for $\lambda$ is larger in this case.

ical charge indices obtained by using different operators do not always agree on a configuration by configuration basis. It is only when one is close to the continuum limit that the results agree. Even with the overlap operator previous studies [12] showed that the topological indices do not agree on a configuration by configuration basis when, for example, a different mass parameter is used in

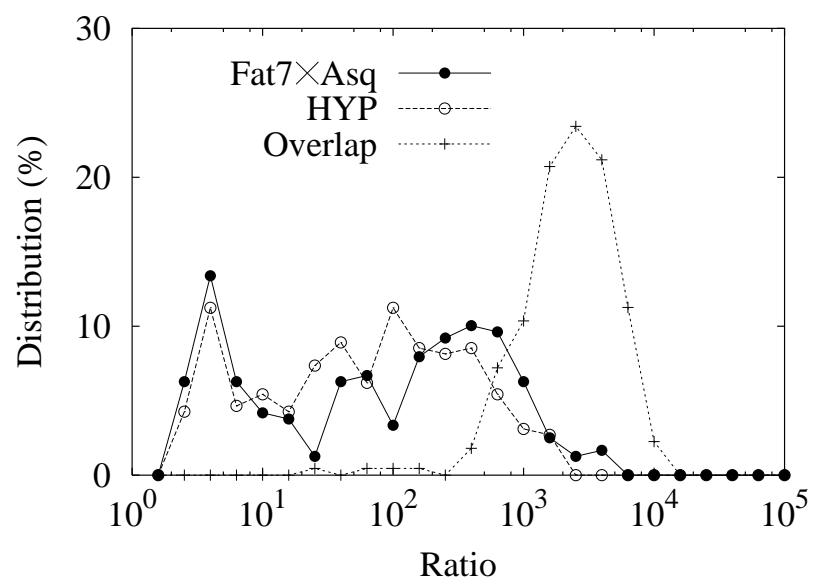

FIG. 5: Same as Fig. 2 but with improved gauge field action $\left(\beta=8.26(a \simeq 0.121 \mathrm{fm}), V=10^{4}\right)$.

the kernel. In the present case, we find that the topological charge indices determined by the different operators agree about $60-70 \%$ of the time, compared to $28 \%$ if the values were completely random. More explicitly, indices from different operators are compared pairwise and the agreement is $63 \%$ for overlap and UFat7 $\times$ Asq, $68 \%$ for overlap and HYP, $78 \%$ for UFat7 $\times$ Asq and HYP. Note that the agreement between UFat7 $\times$ Asq and HYP is larger than that obtained for an improved staggered operator and the overlap operator. It is then important to check whether the charge distributions are also different because physical observables, e.g., the topological susceptibility, are related to the ensemble average of the topological charge. The distributions obtained by these operators are given in Fig. 3. It can be observed that there is no significant difference among the results. This is important because it indicates that physics is independent of the discretization scheme and one would expect topological quantities obtained by these operators to agree.

\section{B. Improvement in the Gauge Field Action}

Lattice artifacts can be further suppressed if improvement is also applied to the gauge field action. This can be seen in Fig. 4 where the infrared eigenvalue spectra of the different staggered operators are shown for configurations generated using the tadpole improved gauge field action. Here $\beta=8.26(a \sim 0.121 \mathrm{fm})$ and lattice volume is $V=10^{4}$ so that both the lattice spacing and physical volume are very similar to those used in the unimproved case. Results here show that better topological properties are realized when the gauge field action is also improved. In particular even the Asqtad operator is sensitive to the topology at this coarse lattice spacing and "zero modes" can be identified unambiguously for the UFat7 $\times$ Asq and HYP operators.

To have a quantitative picture of how improvement in 


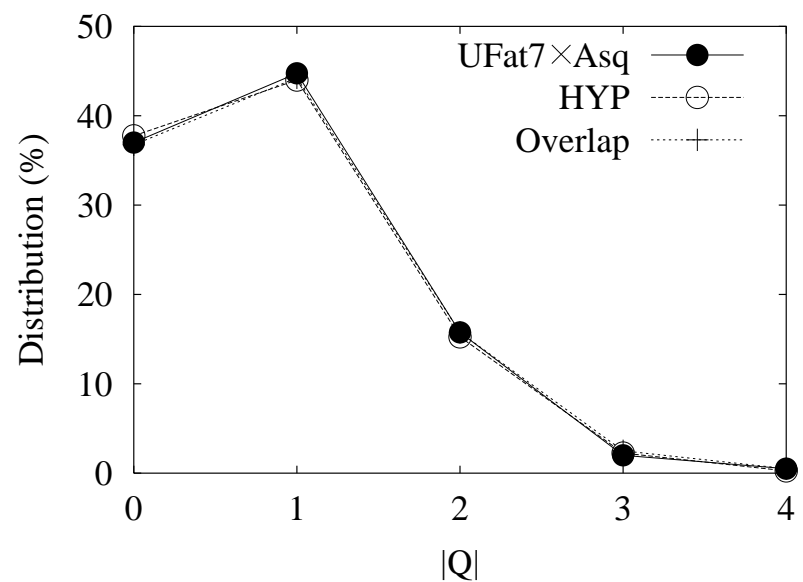

FIG. 6: Same as Fig. 3 but with improved gauge field action $\left(\beta=8.26(a \simeq 0.121 \mathrm{fm}), V=10^{4}\right)$.

the gauge field action affects the spectral flow, we again plot the distribution of the ratios of eigenvalues between the smallest non-chiral mode and the largest "zero mode" in Fig. 5 for configurations with non-trivial topology. The same scale is used in Fig. 2 and Fig. 5 . In comparison to Fig. 22 it can be observed that, for UFat $7 \times$ Asq and HYP, the distribution has shifted significantly toward ratios of two or three orders of magnitude. Overall, more than $95 \%$ of the configurations have ratios $\geq 5$, compared to only $85 \%$ in the case with unimproved gauge fields. Hence gauge field improvement clearly increases the separation between the chiral "zero modes" and the non-chiral modes. We should again emphasize here that the ratios should be infinite theoretically for the overlap operator since exact zero modes exist on the lattice.

We have also compared the topological indices obtained by the different operators in this case. It is found that the agreement increases significantly when the gauge field action is also improved: $91 \%$ between overlap and UFat $7 \times$ Asq, $90 \%$ between overlap and HYP and $96 \%$ between UFat7 $\times$ Asq and HYP which is again the highest. The charge distributions are given in Fig. [6 It can be observed upon comparing with Fig. [3] that better agreement is obtained with improved gauge fields. Results here are significant because they indicate that different operators do respond the same way to the topology of the background gauge fields when discretization errors and lattice artifacts are reduced. In particular, results here show that the charge indices obtained by using the staggered operators and the overlap operator, two completely different representations of the Dirac operator on the lattice, agree even on a configuration by configuration basis at a high percentage as the level of improvement increases.

\section{Dependence on Lattice Spacing}

Here we examine the dependence of the infrared eigenvalue spectrum on lattice spacing and study the spectral flow as one approaches the continuum limit. Calculations are done at three lattice spacings with fixed physical volume $V \sim(1.2 \mathrm{fm})^{4}$ (so lattice volume increases as lattice spacing decreases, see Table【. Results are shown for the Asqtad and UFat7 $\times$ Asq operators in Fig. 7. One sees that separation between the "zero modes" and non-chiral modes becomes more clear. In addition the continuum 4fold degeneracy is better realized as one approaches the continuum limit. Note that the chirality of the "zero modes" increases as the lattice spacing decreases and it is larger for the UFat7 $\times$ Asq operator. This gives evidence to the fact that discretization errors and lattice artifacts are indeed responsible for the failure of staggered fermions to be sensitive to gauge field topology on coarse lattices. The chiral "zero modes" associated with the topology of the background gauge fields emerge as one approaches the continuum limit, a conclusion also obtained in [7].

Our results also show that it is necessary to use a lattice spacing $a \lesssim 0.1 \mathrm{fm}$ for the Asqtad operator to be sensitive to gauge field topology. This is similar to the lattice spacings used nowadays in dynamical simulations of QCD using the Asqtad fermion action and the tadpole improved gauge field action [19]. On the other hand, further improvement of the staggered quark action, e.g, actions with unitarized fat-links UFat7 $\times$ Asq, may be necessary when working on coarse lattices to ensure that continuum physics is reproduced correctly.

\section{Dependence on Lattice Volume}

As discussed in the introduction, we expect a strong dependence of the spectral density on the volume $V$ of the lattice and the topological charge average $\left\langle Q^{2}\right\rangle$ should scale with $V$. In Fig. 8 the infrared eigenvalue spectrum of the UFat $7 \times$ Asq operator is given for lattice volumes $V=8^{4}, 10^{4}, 12^{4}$ and $16^{4}$ at a fixed lattice spacing $a=0.121 \mathrm{fm}(\beta=8.26)$ with improved gauge fields. Results from previous sections showed that "zero modes" should be visibly separated from the non-chiral modes at this spacing for configurations generated by the improved gauge action. This is the case when the volume is $\sim(1.2 \mathrm{fm})^{4}$. A similar volume effect has been seen with the overlap fermion operator also [11, 12]. A reasonable assumption is that this minimum volume effect is related to a property of the gauge field, perhaps some minimum volume is necessary before the topological structure is fully formed.

As the lattice volume increases the number of eigenvalues increases and one may expect the gap between the zero modes and the non-chiral modes to scale as $V^{-1}[29]$. The net result is that there are more low-lying non-chiral modes as volume gets bigger. As Fig. 8 shows, a certain 

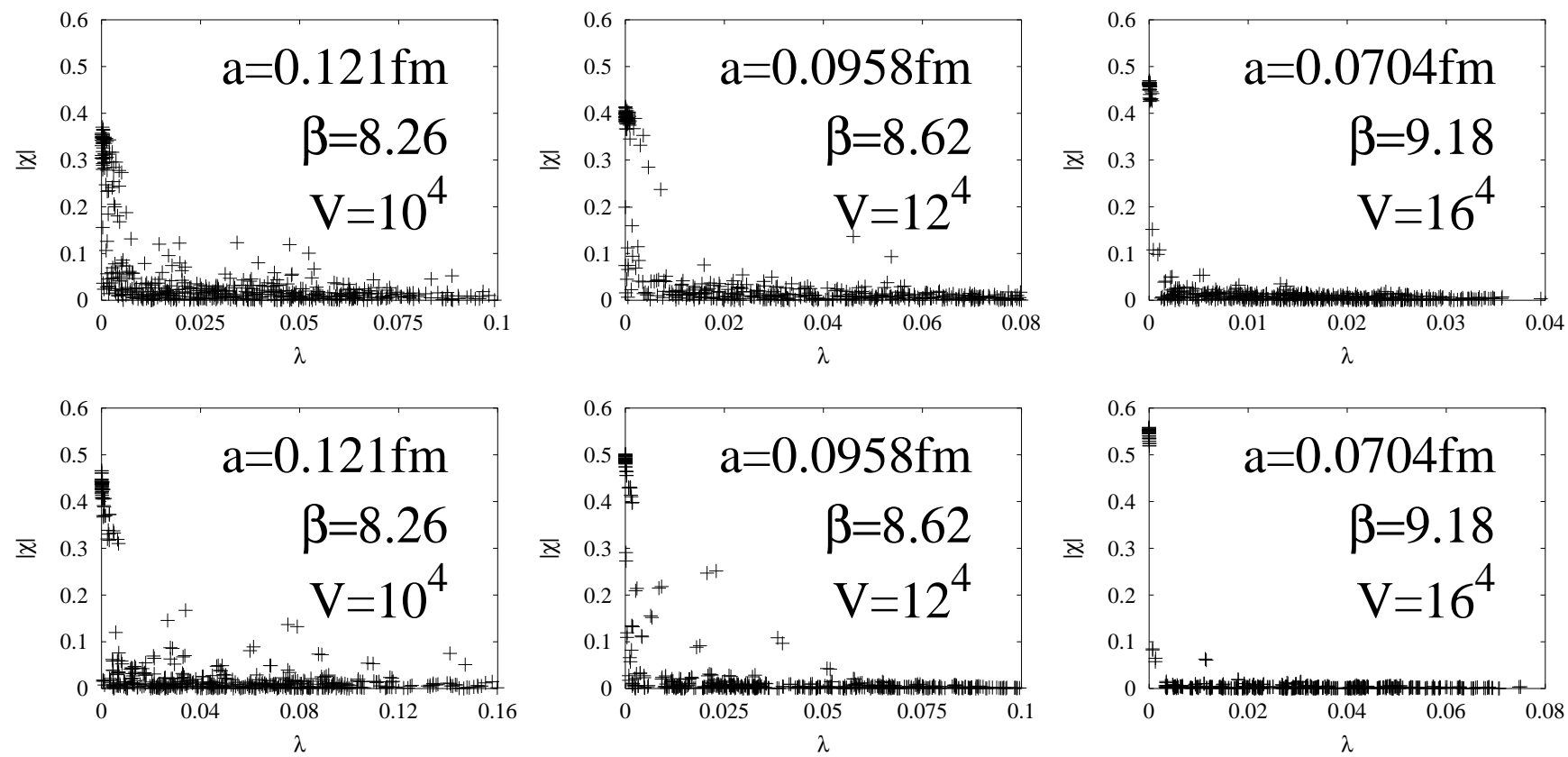

FIG. 7: Dependence on lattice spacing. Results are shown for the Asqtad (top) and the UFat7 $\times$ Asq (bottom) operators with improved gauge fields. Physical volumes are $\sim(1.2 \mathrm{fm})^{4}$ in all cases.
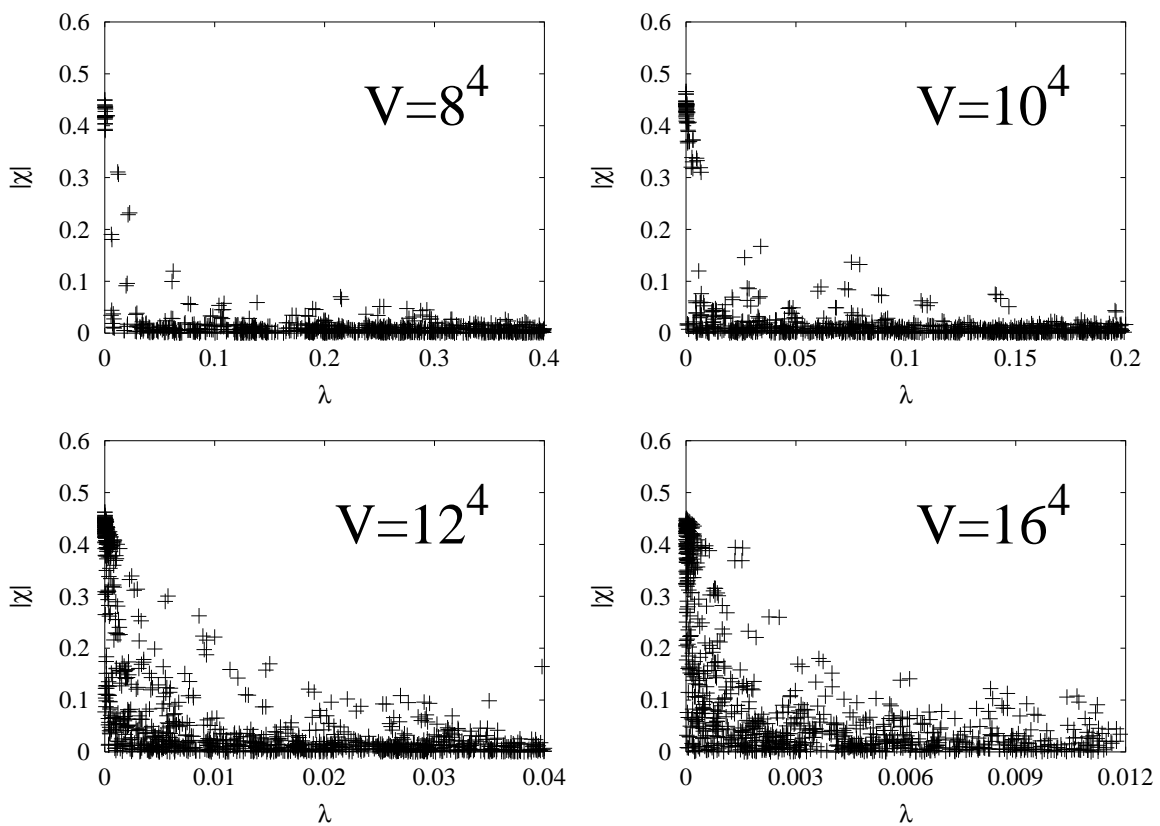

FIG. 8: Spectral graphs for the UFat7 $\times$ Asq operator at a fixed coupling $\beta=8.26(a \simeq 0.121 \mathrm{fm})$ for volumes $V=8^{4}, 10^{4}, 12^{4}$ and $16^{4}$ with improved gauge fields. The spectrum becomes noisy again for large volumes. Note that the scale for $\lambda$ decreases with increasing volume.

volume is necessary before the would-be "zero modes" show up and sensitivity to topology is established. As the volume is increased even further the number of chiral "zero modes" increases roughly as $\sqrt{V}$ as can be inferred from Fig. 9 In the configuration average, the tail of the chiral-mode distribution begins to merge with the low eigenvalue tail of the non-chiral mode distribution. How- ever, the indentification of the would-be "zero modes" is not quite as difficult as it may appear from Fig. 8. As seen from Fig. 9 the criteria which we adopt for a "zero mode", namely, at least a factor of 2 smaller in eigenvalue than the smallest non-chiral mode $\left(R_{\lambda}=2\right)$ and a factor of 5 larger in chirality $\left(R_{\chi}=5\right)$, are very robust even for the $16^{4}$ lattice. Imposing other values for these 


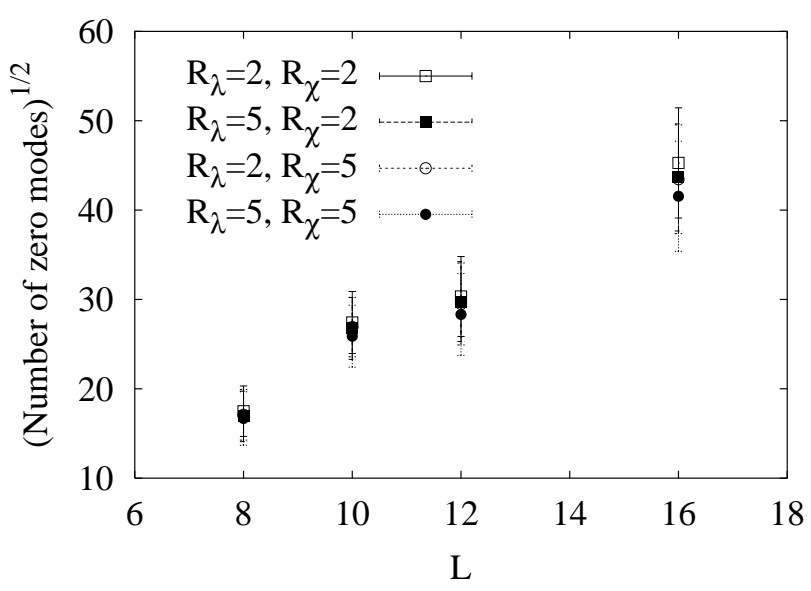

FIG. 9: The square-root of the total number of zero modes of the UFat $7 \times$ Asq operator as a function of lattice size $L$ (lattice volume $V=L^{4}$ ). Results of using different criteria for identifying the zero modes are shown.

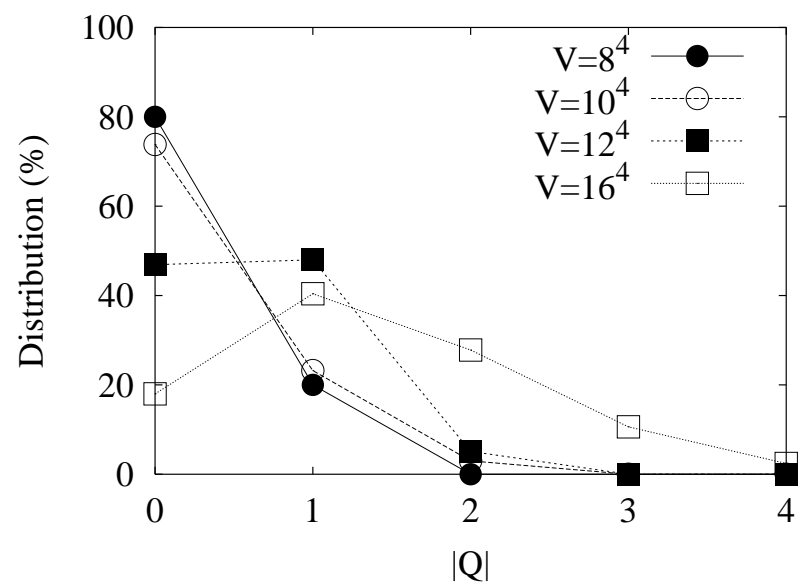

FIG. 10: Topological charge distribution (as a percentage of configurations) for different lattice volumes. Results are shown for the UFat7 $\times$ Asq operator at $\beta=8.26(a \simeq$ $0.121 \mathrm{fm}$ ) with improved gauge fields.

factors gives very similar results.

The expected increase of $\langle|Q|\rangle$ with the volume of the lattice can be seen in Fig. 10] where the topological charge distribution is shown for different lattice volumes. Most configurations have trivial topological structure when the volume is small and the charge average $\langle|Q|\rangle$ increases gradually with the volume of the lattice.

\section{COMPARISON WITH RMT}

Results from our systematic analysis give strong indications that staggered fermions are sensitive to gauge field topology when lattice artifacts are sufficiently suppressed. It is then important to compare the distribution of the non-chiral modes, after successfully identifying the would-be zero modes, against the predictions of RMT. In particular, we study the cumulative distribution of the smallest non-chiral modes in different topological sectors $P_{c u m m}^{(Q)}(\zeta)$ where RMT gives the predictions [5, 30]

$$
\begin{aligned}
P_{\text {cumm }}^{(Q)}(\zeta) & =\frac{\int_{0}^{\zeta} P^{(Q)}(x) d x}{\int_{0}^{\infty} P^{(Q)}(x) d x} \\
P_{\text {cumm }}^{(0)}(\zeta) & =\frac{1}{2} \int_{0}^{\zeta} x e^{-x^{2} / 4} d x \\
P_{\text {cumm }}^{(1)}(\zeta) & =\frac{1}{2} \int_{0}^{\zeta} I_{2}(x) e^{-x^{2} / 4} d x \\
P_{\text {cumm }}^{(2)}(\zeta) & =\frac{1}{2} \int_{0}^{\zeta}\left[I_{2}(x)^{2}-I_{1}(x) I_{3}(x)\right] e^{-x^{2} / 4} d x .(9)
\end{aligned}
$$

Here, $P^{(Q)}(x)$ denotes the distribution of the smallest non-chiral modes in charge sector $Q$ and $I_{n}(x)$ is the modified Bessel function of the first kind (order $n$ ). The variable $\zeta \equiv \lambda \Sigma V$ is related to the infinite-volume chiral condensate $\Sigma$ which is a fitting parameter.

Calculations are done for three different operators $(($ UFat7 $) \times$ Asq, HYP and overlap $)$ on two ensembles of configurations (unimproved and improved gauge fields with $\left.a \sim 0.12 \mathrm{fm}, V=10^{4}\right)$. These ensembles are specifically chosen because the size of the lattice is limited by the cost in computing the eigenvalue spectra of the overlap operator while a coarse grid is used so that the physical volume is larger than the critical value $(1.2 \mathrm{fm})^{4}$. This criterion is important as it ensures that we are in the $\epsilon$-regime where RMT is applicable [11].

In our analysis, the would-be zero modes are first identified and the configurations are classified according to their charge indices obtained using the index theorem (11). We should re-emphasize here that the indices obtained by using different operators do not always agree on a configuration by configuration basis but the charge distributions are indistinguishable (see Fig. B] and 6). It should also be noted that all configurations are used in our analysis. This is different from some previous studies $[5,[6]$ where a small portion of the ensemble was discarded. In these studies the topological charge indices were also calculated using a direct discretization of the continuum formula, $Q_{g} \sim \int \epsilon^{\mu \nu \rho \sigma} F_{\mu \nu} F_{\rho \sigma}$. Only those configurations with approximate integer value of $Q_{g}$ were included in the analysis in Ref. [5], and configurations for which the charge index obtained by the index theorem is different from $Q_{g}$ were excluded in Ref. [6] .

Results are shown in Fig. 11] The solid curves are predictions from RMT. Because computing the spectra for the overlap operator is much more expensive, the sizes of the ensembles are different: 1000 configurations for $($ UFat7) $\times$ Asq and HYP but only 400 for the overlap operator. This is the reason that the overlap results appear to have poorer agreement with the predictions of RMT. Note that the agreement is better with improved gauge fields. The results given here contribute to the evidence that staggered fermions do feel gauge field 

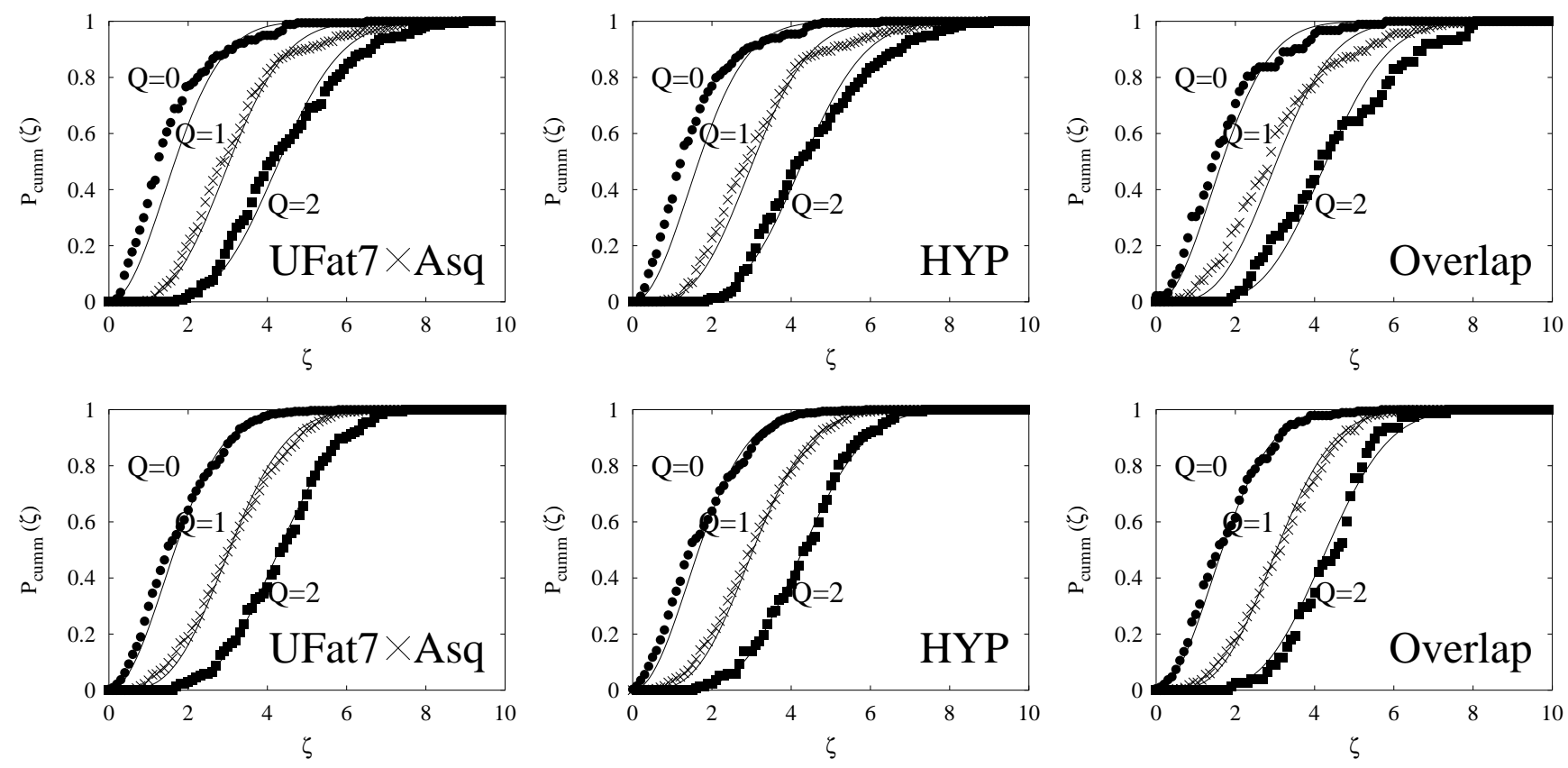

FIG. 11: Cumulative distribution of the smallest non-chiral modes in different topological sectors. Results are shown for unimproved (top) and improved (bottom) gauge fields at $a \sim 0.12 \mathrm{fm}, V=10^{4}$. Solid curves are predictions of RMT.

topology, provided that lattice artifacts are suppressed considerable, as the agreement with the predictions of RMT is impressive. They should be compared with previous studies [4, 5] with the unimproved staggered operator which appeared to indicate the presence of only a trivial topological sector.

\section{CONCLUSION}

In this project we studied numerically the spectral properties of a variety of improved staggered Dirac operators. Four systematics have been examined: i) improvement in the staggered operator, ii) improvement in the gauge field action, iii) lattice spacing and iv) lattice volume.

It has been observed that the infrared eigenvalue spectrum depends sensitively on the way in which the staggered fermion operator is improved. On coarse lattices the unimproved operator is insensitive to gauge field topology. As the level of improvement increases, either on the operator itself or the background gauge fields, eigenmodes with small eigenvalues and large chirality appear. These small eigenmodes can be identified as the chiral "zero modes" associated with the topology of the gauge fields. Sensitivity to the topology also increases as one approaches the continuum limit. This gives evidence that lattice artifacts are responsible for the failure of the unimproved staggered operator to reflect properly the gauge field topology on coarse lattices. Our results also show that a lattice spacing $a \lesssim 0.1 \mathrm{fm}$ is enough for the Asqtad operator to have a correct response to the topology with improved gauge fields. This spacing is of the order of the lattice spacings used in present day state-of-the-act dynamical simulations of QCD. On the other hand, the next level of improved staggered operators, e.g., UFat7 $\times$ Asq, may be required to produce configurations which describe the correct continuum physics on coarser lattices. We also observe that the topological charge distribution is independent of which operator is used even though the charge indices do not always agree on a configuration by configuration basis. However, the agreement increases with the level of improvement. A minimum physical volume of about $(1.2 \mathrm{fm})^{4}$ seems to be necessary in order for "zero modes" to show up and for sensitivity to topology to be established. This effect 11, 12 was observed earlier for overlap fermions. As volume is increased the number of would-be "zero modes" increases as $\sqrt{V}$ but the number of low-lying non-chiral modes increases faster so some merging of tails of the distributions takes place. Up to the $16^{4}$ volume considered here it is still possible to make a clear separation of would-be chiral modes from nonchiral modes. The distribution of the non-chiral modes is matched with the predictions of RMT. The agreement is comparable to that obtained using overlap fermions.

Based on this, and other work [6, 7] done in the past year, one has strong evidence that, provided one uses improved staggered operators and improved gauge fields, staggered fermions properly feel gauge field topology. 


\section{Acknowledgments}

We are very grateful to J. B. Zhang for providing the eigenvalue solver for the overlap operator and $\mathrm{H}$. D. Trottier for many useful discussions. The computa- tions were performed on facilities provided by WestGrid (http://www.westgrid.ca/home.html). This work was supported in part by the Natural Sciences and Engineering Research Council of Canada.
[1] M. F. Atiyah and I. M. Singer, Ann. Math. 87, 484 (1968); 93, 119 (1971).

[2] See, for example, K. Jansen, Nucl. Phys. B (Proc. Suppl.) 129, 3 (2004).

[3] M. E. Berbenni-Bitsch, S. Meyer, A. Schäfer, J. J. M. Verbaarschot, and T. Wettig, Phys. Rev. Lett. 80, 1146 (1998); M. E. Berbenni-Bitsch, S. Meyer, and T. Wettig, Phys. Rev. D 58, 071502 (1998).

[4] M. Göckeler, H. Hehl, P. E. L. Rakow, A. Schäfer, and T. Wettig, Phys. Rev. D 59, 094503 (1999).

[5] P. H. Damgaard, U. M. Heller, and A. Krasnitz, Phys. Lett. B 445, 366 (1999); P. H. Damgaard, U. M. Heller, R. Niclasen, and K. Rummukainen, Phys. Rev. D 61, 014501 (2000).

[6] E. Follana, A. Hart, and C.T.H. Davies, Phys. Rev. Lett. 93, 241601 (2004).

[7] S. Dürr, C. Hoelbling, and U. Wenger, Phys. Rev. D 70, 094502 (2004). See also S. Dürr and C. Hoelbling, Phys. Rev. D 69, 034503 (2004).

[8] K. Y. Wong and R. M. Woloshyn, hep-lat/0407003

[9] F. Farchioni, I. Hip, and C. B. Lang, Phys. Lett. B 471, 58 (1999).

[10] J.B. Kogut, J.F. Lagaë, and D.K. Sinclair, Phys. Rev. D 58, 054504 (1998).

[11] W. Bietenholz, K. Jansen, and S. Shcheredin, JHEP 0307, 33 (2003); L. Giusti, M. Lüscher, P. Weisz, and H. Wittig, JHEP 0311, 23 (2003).

[12] J. B. Zhang, et al., Phys. Rev. D 65, 074510 (2002).

[13] T. Kalkreuter, Phys. Rev. D 51, 1305(1995).

[14] H. Neuberger, Phys. Lett. B 417, 141 (1998); Phys. Rev. Lett. 81, 4060 (1998).

[15] M. Lüscher, Phys. Lett. B 428, 342 (1998).
[16] G.P. Lepage, Phys. Rev. D 59, 074502 (1999).

17] MILC Collaboration, C. Bernard et al., Phys. Rev. D 58, 014503 (1998).

[18] K. Orginos, D. Toussaint, and R.L. Sugar, Phys. Rev. D 60, 054503 (1999).

[19] See, for example, C. T. H. Davies et al., Phys. Rev. Lett. 92, 022001 (2004); C. Bernard et al., Phys. Rev. D 68, 114501 (2003).

[20] W. Lee, Phys. Rev. D 66 (2002) 114504.

[21] HPQCD Collaboration, E. Follana et al., Nucl. Phys. B (Proc.Suppl.) 129, 447 (2004).

[22] A. Hasenfratz and F. Knechtli, Phys. Rev. D 64, 034504 (2001).

[23] R. G. Edwards, U. M. Heller, and R. Narayanan, Nucl. Phys. B535, 403 (1998).

[24] P. H. Ginsparg and K. G. Wilson, Phys. Rev. D 25, 2649 (1982).

[25] E. I. Zolotarev, Zap. Imp. Akad. Nauk. St. Petersburg, 30, 5 (1877); reprinted in Collected Works (Akademii Nauk SSSR, Moscow, 1932), Vol. 2, pp.1-59; see also T. W. Chiu, T. H. Hsieh, C. H. Huang, and T. R. Huang, Phys. Rev. D 66, 114502 (2002).

[26] F. Okiharu and R. M. Woloshyn, Eur. Phys. J. C35, 537 (2004).

[27] M. F. L. Golterman and J. Smit, Nucl. Phys. B245, 61 (1984).

[28] J. Smit and J. C. Vink, Nucl. Phys. B286, 485 (1987).

[29] S. J. Hands and M. Teper, Nucl. Phys. B347, 819 (1990).

[30] J. J. M. Verbaarschot and I. Zahed, Phys. Rev. Lett. 70, 3852 (1993). 